\title{
Genders in the Life Course: Demographic Issues.
}

\section{Edited by Antonella Pinnelli, Filomena Racioppi, and Rosella Rettaroli}

Dordrecht, The Netherlands: Springer, 2007. ISBN: 978-1402060014, \$186.62, 328 pages.

\author{
Susan A. McDaniel \\ University of Utah \\ susan.mcdaniel@ipia.utah.edu
}

Life course is a perspective whose time has evidently come. It is utilized in widening numbers of social science studies across a vast range of substantive foci. Demography may be a late but welcome arrival to the life course perspective, although in Canada we, of course, have established a Cluster on Population Change and Life Course, which is integrating the demographic and life course perspectives.

This volume, edited by three women demographers and one statistician, all from Italy, is a most welcome contribution to the research literature. The book is comprised of eleven substantive chapters ranging from the macro, such as consideration of gender systems to the micro, i.e., age at first intercourse and ideational factors and choices involved in union formation. These chapters are preceded by an introductory chapter. The research stems from a grant coordinated by Antonella Pinnelli, on gender and demography.

As outlined in the opening pages of the volume, Pinnelli, Racioppi, and Rettaroli aim to provide evidence of the relations between demographic behaviours and gender systems from the perspective of life course. In this effort, they join an impressive number of researchers, one of whom is Hannah Brückner (2004), whose work they do not cite. Acknowledging that gender in demography has, until recently, been largely overlooked except as a categorization scheme, they further argue that gender studies could benefit from the infusion of a demographic perspective, particularly given that many demographic indicators are markers of a gendered system of relations. They refer briefly to four theoretical approaches to gender in demography, the main one being "the new home economics," and then suggest that for much of demographic behaviour, no theory exists that incorporates gender and gender systems, a conclusion with which this reviewer is inclined to 
agree. Data for the substantive chapters come centrally from the Family and Fertility Surveys, begun in Europe in the late 1980s, but including Canada, the U.S. and New Zealand as well as European countries.

The eleven chapters are not organized into sections, but could be in various ways. For example, many deal with some aspect of women's paid employment - in relation to gender systems (Chapter 1, Di Giulio and Pinnelli), in connection with first union formation (Chapter 3, Impicciatore and Rettaroli), and couple choices (Chapter 4, Pasquini and Samoggia), fertility (Chapter 5, Pinnelli and Di Giulio), union dissolution (Chapter 7, De Rose and Di Cesare) and its aftermath (Chapter 8, Angeli and De Rose), and migration (Chapter 9, Farina and Terzera). In all aspects and transitions, women's employment is found to be significantly related to choices made. In countries with a family-oriented culture, marriage or union formation is part of the achievement of enhanced gender symmetry and the reduction of subjection in the relationship. In eastern European countries, by contrast, where a pattern of women's employment has long existed, a substantial degree of independence between the family and work emerges from analyses here. The editors suggest, based on the findings here, that women's labour market participation may well be seen as a contributing element to making gender a social construct. Women's increased autonomy, gained rapidly in several of the countries studied, alters the bases of gender relations both in family and in the public sphere, thereby altering the gender systems and demographic behaviours.

Another way in which the eleven chapters might be organized would be by micro or macro themes. While the individual perspective prevails in this volume, macrolevel approaches treat gender as a dimension of social systems. This is particularly evident in Chapter 1 (by Di Guilio and Pinnelli) where multiple methods are utilized to spotlight the temporal dynamics of gender systems. They find, in detailed analyses of time use, of divisions of labour in couples, and other dimensions of change over time, that clear gender systems exist that intersect with demographic behaviours and help to explain changes in both. Specifically, four gender systems emerge: 1) an eastern European one where primacy is given to the economic sphere with ostensible public gender equality but less equality in couples; 2) a Nordic countries system with both public and private gender equality, at least to some extent; 3) a Southern European system with primacy on family and household tasks for women; and 4) the system of Northern and Western Europe where women's labour market participation is not as high as in Eastern Europe but women are more extensively involved in the public sphere.

This edited book and all its chapters are heavily empirical. There is mention of theoretical framing and the need for theory that unites gender 
and demographic perspectives via the life course. And yet, ultimately, there is little theory offered.

That said, the research here contributes to our understanding of life course, by examining demographic life course transitions as situated in gender systems of relations. In that, it makes an important contribution. Helpful in the volume would have been a summary chapter by the editors, taking the reader back to what the aims of the book were, developing on the basis of the empirical findings here, a multilevel conceptual framework, a mesotheory perhaps, of gender in the life course through demographic behaviours.

This book could grace any sociologist's book shelf. It would strongly benefit demographers to be exposed to these fine-grained analyses, particularly in North America. It is also useful to comparative international research. The sophistication of the analytical approaches would make it difficult for use in most (many) undergraduate courses, but perhaps could be useful in a senior demography course, or certainly in a graduate course.

\section{References}

Brückner, Hannah. 2004. Gender Equality in the Life Course: Social Change and Stability in West Germany, 1975-1995. Hawthorne, NY: Aldine de Gruyter. 